\title{
Correction to: Adverse Drug Reaction Case Safety Practices in Large Biopharmaceutical Organizations from 2007 to 2017: An Industry Survey
}

\author{
Stella Stergiopoulos ${ }^{1}$ (1) $\cdot$ Moritz Fehrle $^{2} \cdot$ Patrick Caubel $^{3} \cdot$ Louise Tan $^{4} \cdot$ Louise Jebson $^{4}$
}

Published online: 18 December 2019

(c) Springer Nature Switzerland AG 2019

Correction to: Pharmaceutical Medicine (2019) 33:499-510
https://doi.org/10.1007/s40290-019-00307-x

The correct name of the second author should be "Moritz Fehrle", and not "Mortiz Fehrle" as given in the original publication of the article.

Correction to Supplementary Material:

The correct name of the second author should be "Moritz Fehrle", and not "Mortiz Fehrle" as given in the original publication of the article.

The original article can be found online at https://doi.org/10.1007/ s40290-019-00307-x.

Electronic supplementary material The online version of this article (https://doi.org/10.1007/s40290-019-00319-7) contains supplementary material, which is available to authorized users.

Stella Stergiopoulos

Stella.Stergiopoulos@tufts.edu

1 Tufts Center for the Study of Drug Development, Tufts University School of Medicine, 75 Kneeland Street, Ste 1100, Boston, MA 02111, USA

2 Bayer AG, 51368 Leverkusen, Germany

3 Pfizer Inc, New York, NY 10017, USA

4 Pvnet ${ }^{\circledR}$, Navitas Life Sciences GmbH, 60528 Frankfurt, Germany 